\title{
NICE guidelines on the menopause
}

\author{
Missing quantitative summary estimates of risks of hormone therapy
}

\author{
Martha Hickey professor of obstetrics and gynaecology ${ }^{1}$, Emily Banks professor of epidemiology \\ and public health ${ }^{2}$
}

'Department of Obstetrics and Gynaecology, University of Melbourne and the Royal Women's Hospital, Victoria, Australia ; ${ }^{2}$ National Centre for Epidemiology and Population Health, Research School of Population Health, Australian National University, Canberra, ACT 2601, Australia

The first National Institute for Health and Clinical Excellence guidelines on menopause appropriately highlight how symptoms may impair quality of life and function and persist for many years for some women. ${ }^{1}$ The guidelines promote individualised care and emphasise the need for adequate clinical services and further research.

While the NICE guidelines emphasise the clear evidence that menopausal hormone therapy (MHT, often referred to as hormone replacement therapy) provides highly effective relief of symptoms, there is less focus on other useful options. Recent high quality evidence shows that selected antidepressants are equivalent to very low dose oestrogen for vasomotor symptoms and sleep, ${ }^{23}$ and gabapentin has a similar efficacy. ${ }^{4}$ For women who want to self manage, techniques such as yoga and cognitive behavioural therapy may reduce symptoms and also improve quality of life and mood. ${ }^{5}{ }^{6}$ Women should not be "suffering in silence" but should be informed about alternatives to MHT, even if these are not as effective, and the risks and benefits of MHT so that they can make an informed choice.

Importantly, despite the rigorous process of the NICE guideline development, methodological deficiencies undermine its conclusions. Specifically, the guideline lacks appropriate and complete quantitative summary estimates of the risks and benefits from taking MHT. ${ }^{7}$ Estimates of relative risk should be calculated from all relevant randomised controlled trials for cardiovascular disease outcomes and from trial and observational data for cancer. These estimates should then be used to calculate absolute risk, including estimates based on the combined current evidence regarding risk according to duration of use. ${ }^{7}$ Instead of such summaries, the guidelines are likely to cause confusion by presenting multiple risk estimates from selected studies, often with overlapping confidence intervals or stating that there are "no data available" even where estimates could be calculated. The focus is on non-significant risk estimates in the 50-59 age subgroup, rather than estimating absolute risk from the best available data. Because the relative risks of disease related to MHT do not vary significantly by age (except that breast cancer risk may be greater with use closer to menopause ${ }^{8}$ ) they should be quoted for all age groups combined. ${ }^{7}$ The lack of a significant increase in risk in an underpowered age subgroup is not robust evidence of safety or of "no effect."

The clinical challenge for MHT is balancing the benefits of symptomatic relief against the risks of disease. The guidelines were largely reassuring about safety and suggest potential benefits for long term health. However, current users are at increased risk of venous thromboembolism, stroke, breast cancer, and potentially ovarian cancer. Risks are greater with combined MHT, rise with duration of use, and fall on cessation. ${ }^{7}$ The drop in use of MHT following greater awareness of risk was accompanied by substantial reductions in incidence of breast cancer. ${ }^{9}{ }^{10}$ Women may also want to know that systemic MHT may increase urinary incontinence, ${ }^{11}$ gallbladder disease, and dementia. ${ }^{12}$

Limited data suggest that oestrogen may confer health benefits in women with early menopause. However, the search for long term overall health benefits of MHT in women at the normal age of menopause has largely been fruitless. International drug regulatory agencies do not support its use for long term health, instead recommending MHT for troublesome menopausal symptoms, for as short a time as possible. ${ }^{713}$ However, recurrence of symptoms on discontinuation is common.

The NICE guidelines advise women to avoid MHT after breast cancer and, despite alluding to the safety of newer selective oestrogen receptor modulators in this population, no published human data are cited. Women without breast cancer who are at increased genetic risk of breast and ovarian cancer are also advised to avoid MHT. Risk reducing bilateral oophorectomy before menopause is advised for these women and may lead to severe menopausal symptoms. The authors may wish to clarify whether MHT is contraindicated in these women and why.

It was refreshing to see a research agenda for menopause but disappointing that four out of five items focused on MHT. There are cavernous gaps in the science of menopause: What regulates menopause timing? What is the mechanism of vasomotor symptoms? What causes sleep and mood disturbance? The research agenda is optimistic that further studies of new MHT 
formulations will show they have greater safety. Given the number of large and costly trials already completed, the justification for further studies seems uncertain and a broader research agenda would be welcome.

Society has strong negative perceptions of female ageing. Wider acceptance that menopause is a normal transition rather than an "oestrogen deficiency syndrome" might be more empowering for women. Women and their healthcare providers should have confidence that most will manage their symptoms without pharmacotherapy. The UK Medicines and Healthcare Products Regulatory Agency estimates that five years' use of combined MHT results in an excess of serious, potentially life threatening disease (obtained by adding the estimated number of extra cases of breast cancer, ovarian cancer, stroke, coronary heart disease, and venous thromboembolism likely to be caused by MHT and subtracting the number of fractures and colorectal cancers prevented) in $1.4 \%$ of women aged $50-59$ and $2.2 \%$ of those in their $60 \mathrm{~s} .{ }^{7}$ Whether such risks outweigh the benefits is for women themselves to decide, supported by the highest quality evidence and by their health professionals.

Competing interests: We have read and understood BMJ policy on declaration of interests and have no relevant interests to declare.

Provenance and peer review: Commissioned; not externally peer reviewed.
1 National Institute for Health and Care Excellence. Menopause: diagnosis and management of menopause. (NICE guideline 23.) 2015. www.nice.org.uk/guidance/ng23.

2 Guthrie KA, LaCroix AZ, Ensrud KE, et al. Pooled analysis of six pharmacologic and nonpharmacologic interventions for vasomotor symptoms. Obstet Gynecol 2015;126:413-22.

3 Ensrud KE, Guthrie KA, Hohenseem C, et al. Effects of estradiol and venlafaxine on insomnia symptoms and sleep quality in women with hot flashes. Sleep 2015;38:97-108.

4 Bordeleau L1, Pritchard KI, Loprinzi CL, et al. Multicenter, randomized, cross-over clinical trial of venlafaxine versus gabapentin for the management of hot flashes in breast cancer survivors. J Clin Oncol 2010;28:5147-52.

5 Mann E, Smith MJ, Hellier J, et al. Cognitive behavioural treatment for women who have menopausal symptoms after breast cancer treatment (MENOS 1): a randomised controlled trial. Lancet Oncol 2012;13:309-18.

6 Reed SD, Guthrie KA, Newton KM, et al. Menopausal quality of life: RCT of yoga, exercise, and omega-3 supplements. Am J Obstet Gynecol 2014;210:244.e1-11.

7 Medicines and Healthcare Products Regulatory Authority. Hormone replacement therapy: safety update. MHRA, 2007.

8 Prentice RL, Manson JE, Langer RD, et al. Benefits and risks of postmenopausal hormone therapy when it is initiated soon after menopause. Am J Epidemiol 2009;170:12-23.

9 Ravdin PM, Cronin KA, Howlader N, et al. The decrease in breast cancer incidence in 2003 in the United States. N Engl J Med 2007;356:1670-4.

10 Canfell K, Banks E, Clements M, et al. Sustained lower rates of HRT prescribing and breast cancer incidence in Australia since 2003. Breast Cancer Res Treat 2009;117:671-3.

11 Cody JD, Jacobs ML, Richardson K, Moehrer B, Hextall A. Oestrogen therapy for urinary incontinence in post-menopausal women. Cochrane Database Syst Rev 2012;10:CD001405.

12 Shumaker SA, Legault C, Kuller L, et al. Conjugated equine estrogens and incidence of probable dementia and mild cognitive impairment in postmenopausal women: Women's Health Initiative Memory Study. JAMA 2004;291:2947-58.

13 FDA. Guidance for industry. Noncontraceptive estrogen drug products for the treatment of vasomotor symptoms and vulvar and vaginal atrophy symptoms-recommended prescribing information for health care providers and patient labeling. Draft guidelines. Revision 4. 2005. www.fda.gov/downloads/drugs/guidancecomplianceregulatoryinformation guidances/ucm075090.pdf.

Cite this as: BMJ 2016;352:1191

C) BMJ Publishing Group Ltd 2016 